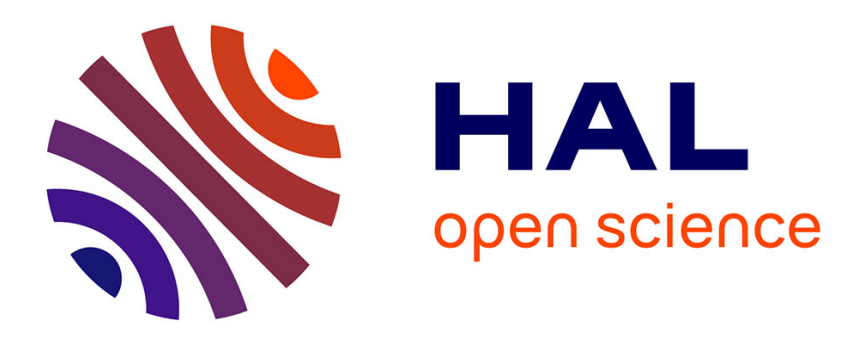

\title{
Random walk in quasi-periodic random environment Julien Brémont
}

\section{To cite this version:}

Julien Brémont. Random walk in quasi-periodic random environment. Stochastics and Dynamics, 2009, 9 (1), pp.47-70. hal-00794132

\section{HAL Id: hal-00794132 \\ https://hal.science/hal-00794132}

Submitted on 25 Feb 2013

HAL is a multi-disciplinary open access archive for the deposit and dissemination of scientific research documents, whether they are published or not. The documents may come from teaching and research institutions in France or abroad, or from public or private research centers.
L'archive ouverte pluridisciplinaire HAL, est destinée au dépôt et à la diffusion de documents scientifiques de niveau recherche, publiés ou non, émanant des établissements d'enseignement et de recherche français ou étrangers, des laboratoires publics ou privés. 


\title{
RANDOM WALK IN QUASI-PERIODIC RANDOM ENVIRONMENT
}

\author{
Julien Brémont \\ Université Paris-Est, mars 2008
}

\begin{abstract}
We consider a one-dimensional random walk with finite range in a random medium described by an ergodic translation on a torus. For regular data and under a Diophantine condition on the translation we prove a central limit theorem with deterministic centering.
\end{abstract}

\section{Introduction}

This paper deals with a model of finite range random walk on $\mathbb{Z}$ in random environment. The set of possible jumps is assumed to be a fixed bounded interval, independent of the point. We first describe this model (called the $(L, R)$-model in the sequel) in full generality.

Model. Introduce a set $\Omega$ of "environments" given via a dynamical system $(\Omega, \mathcal{F}, \mu, T)$, where $T: \Omega \rightarrow \Omega$ is invertible, bimeasurable with respect to $\mathcal{F}$ and preserves a probability measure $\mu$. The system is supposed to be ergodic.

Fixing integers $L \geq 1, R \geq 1$, define as set of possible jumps $\Lambda=\{-L, \cdots,+R\} \subset \mathbb{Z}$. To determine the transition laws of the random walk, let positive random variables $\left(p_{z}\right)_{z \in \Lambda}$ on $(\Omega, \mathcal{F})$ be such that for some constant $\varepsilon>0$ :

$$
\forall z \in \Lambda \backslash\{0\}, p_{z} \geq \varepsilon \text { and } \sum_{z \in \Lambda} p_{z}=1, \mu-a e .
$$

For any $\omega \in \Omega$, we define a random walk $\left(\xi_{n}^{\omega}\right)_{n \geq 0}$ on $\mathbb{Z}$ by $\xi_{0}^{\omega}=0$ and the evolution :

$$
\mathcal{P}_{0}^{\omega}\left(\xi_{n+1}^{\omega}=k+z \mid \xi_{n}^{\omega}=k\right)=p_{z}\left(T^{k} \omega\right), k \in \mathbb{Z}, z \in \Lambda
$$

We denote by $\left(\mathcal{P}_{k}^{\omega}\right)_{k \in \mathbb{Z}}$ the canonical Markovian measures on trajectories corresponding to initial points $k \in \mathbb{Z}$. Our point of view is quenched, ie we consider $\left(\xi_{n}^{\omega}\right)_{n \geq 0}$ under $\mathcal{P}_{0}^{\omega}$, for $\mu-a e \omega$.

Background. The analysis of the $(L, R)$-model is notably more delicate than that of the classical nearest-neighbour model (cf the first chapters of Sznitman [27] or Zeitouni [28] for instance). It involves products of random matrices, Lyapunov exponents and (multi)-linear algebra. A detailed exposition of the $(L, R)$-model is provided in [8,9], with a focus on Key's recurrence criterion [16] and the question of the Law of Large Numbers. We briefly recall the picture and next discuss recent results on the CLT (in a quenched sense, unless otherwise stated) in connection with random walks on a strip $\mathbb{Z} \times\{0, \cdots, m-1\}$.

Generalizing the result of Solomon [26], Key [16] gave a recurrence criterion for the random walk, now expressed in terms of the sign of a central Lyapunov exponent of a random square-matrix $M$, built with the $\left(p_{z}\right)_{z \in \Lambda}$ and of size $d:=L+R-1$. An alternative and more algebraic proof

\footnotetext{
AMS 2000 subject classifications : 60F17, 60J10, 60K37, 37A45, 37C55, 37E10.

Key words and phrases : finite range random walk, Central Limit theorem, coboundary equation,

Lyapunov spectrum, translation on a torus.
} 
was presented in [8], where, also, the a priori quite abstract criterion was shown to be effective. Next, as a corollary of [7], the Law of Large Numbers appeared to be always satisfied : there is a constant $c$ such that $n^{-1} \xi_{n}^{\omega} \rightarrow c\left(\mathcal{P}_{0}^{\omega}-a e, \mu-a e\right)$. The purpose of [9] was then to characterize the situation $c \neq 0$, after studying the invariant measure equation for the environments seen from the particle and extending classical results by Conze and Guivarc'h in [11].

Concerning the CLT, mention that its status for the nearest-neighbour model is not the same in the recurrent and transient regimes. In the recurrent situation, the validity of the CLT is equivalent to the existence of a stringent coboundary decomposition $\log \left(p_{1} / p_{-1}\right)=\varphi-\varphi \circ T$ with $\exp |\varphi| \in L^{1}(\mu)$ (see [7] theorem 4.5, proved for the $(L, R)$-model with $\min \{L, R\}=1$ ), for example not likely to be satisfied in an iid-environment where Sinaï's behavior [25] holds. In the transient case the CLT is more natural and versions with various random centerings were recently proved by Goldsheid in [13] for an iid environment (cf also Peterson [22], independently) or for a medium satisfying some uniform ergodicity. For the $(L, 1)$-model and an environment given by an irrational rotation on the Circle, a CLT with deterministic centering was given in [7], largely extending former results by Alili [1] on the nearest-neighbour model.

We now discuss the CLT for the general $(L, R)$-model, in relation with random walks on a strip. This model, introduced by Bolthausen and Goldsheid in [5], is essentially equivalent to the $(L, R)$ model, even if the jumps structure differs in a non-negligeable way (especially when $L \neq R$ ) and the link between corresponding results, for instance recurrence criteria, is indirect. In a following paper [6], the case of a recurrent medium was remarkably settled in the independent case : the walk follows Sinai's regime unless being a martingale (ie with zero local drift), thus satisfying a CLT in this case. The analysis fundamentally uses the iid-character of the medium. In the transient situations and still for an independent environment, a CLT with random centering was shown by Goldsheid in [14]. Media satisfying a mixing condition were investigated by Roitershtein in [24], who proved an annealed CLT for transient walks on a strip. As mentionned in a remark in [14], some more correlated environments could also be treated. More precisely and although not presently proved, it is reasonable (using [13] and exploiting the continuity resulting from uniform convergence arguments) to expect that a CLT with random centering holds true for the strip model in a transient medium defined by a topological and uniquely ergodic dynamical system, when the data are continuous.

Result. In the context of the $(L, R)$-model, the purpose of this text is to furnish a natural class of environments in which a quenched CLT with deterministic centering is valid in any asymptotic regime. This appears interesting in view of the former results on the CLT for the strip model. We consider environments of quasi-periodic type and more precisely the case when the dynamical system is an ergodic translation on a torus, together with regular data and a compatible Diophantine condition on the translation. The exact statement is given below. As detailed later, the analysis principally consists in solving some general coboundary equations. Such resolutions prove directly the CLT in the recurrent case, as for the $(L, 1)$-model, and eliminate the fluctuations of the centering around its mean in the transient cases. The result generalizes theorem 5.7 in [7] obtained when $\min \{L, R\}=1$ and the torus is the Circle. We keep the same strategy, due to Kozlov [18], but observe that the present extension requires much more material, as there is a serious gap between the situation $\min \{L, R\}=1$ and the general case, see [8,9]. This study complements [8, 9].

In the same perspective, mention in passing that another interesting situation (even more realistically modeling quasi-periodic environments) would be to consider the case when the dynamical system is a general and "generic" interval exchange transformation. One may notice that coboundary equations for IET were recently studied by Marmi, Moussa and Yoccoz in [20].

Let us now formulate the theorem. Fixing some $m \geq 1$, we consider as set of environments the $m$-dimensional torus $\Omega=\mathbb{T}^{m}$, identified with $\mathbb{R}^{m} / \mathbb{Z}^{m}$, with a translation $T: x \longmapsto x+\alpha$ and Lebesgue measure $\mu$. The translation is assumed to be ergodic, in other words the components of $\alpha=\left(\alpha_{1}, \cdots, \alpha_{m}\right)$ together with 1 are rationally independent. The type of $\alpha$ is defined by :

$$
\eta(\alpha)=\sup \left\{\eta>0, \quad \liminf _{\|n\|_{\infty} \rightarrow+\infty}\|n\|_{\infty}^{\eta} \operatorname{dist}(\langle n, \alpha\rangle, \mathbb{Z})=0\right\}
$$


where $n=\left(n_{1}, \cdots, n_{m}\right)$ and $\langle n, \alpha\rangle=n_{1} \alpha_{1}+\cdots+n_{m} \alpha_{m}$. It is classical that $\eta(\alpha) \geq m$ and that Lebesgue almost-all $\alpha$ in $\mathbb{T}^{m}$ have type $m$. For example when $m=1$ algebraic irrational numbers have type 1. See Khinchin [17] for instance. We next introduce classes of regularity.

\section{Definition 1.1}

For $r \geq 0$ and $0 \leq s \leq 1$, we say $f: \mathbb{T}^{m} \rightarrow \mathbb{R}$ is $C_{d i r}^{r, s}\left(\mathbb{T}^{m} \rightarrow \mathbb{R}\right)$ if for all $1 \leq i \leq m$ the partial derivatives $\left(\partial^{l} f / \partial x_{i}^{l}\right)_{0 \leq l \leq r}$ continuously exist on $\mathbb{T}^{m}$ and $\partial^{r} f / \partial x_{i}^{r}$ is s-Hölder continuous in the variable $x_{i}$ with a Hölder constant uniform on $\mathbb{T}^{m}$.

The motivation for considering such non-conventional classes of functions is the rate of decay of Fourier coefficients, see the statement of lemma 1.3 below. Observe that if $m=1$, then $C_{d i r}^{r, s}\left(\mathbb{T}^{1} \rightarrow\right.$ $\mathbb{R})$ is just the set of $C^{r}$ maps $f$ such that $f^{(r)}$ is $s$-Hölder continuous. If $m \geq 2$ and $r \geq 1$, then a $C_{d i r}^{r, s}\left(\mathbb{T}^{m} \rightarrow \mathbb{R}\right)$ map $f$ is $C^{1}$ in the classical sense, but may not be $C^{2}$ if $r=2$. On the other hand, if $f$ is $C^{r}$ in the classical way with a $s$-Hölder differential $D^{(r)} f$, then it is $C_{d i r}^{r, s}\left(\mathbb{T}^{m} \rightarrow \mathbb{R}\right)$. The class $C_{d i r}^{r, s}\left(\mathbb{T}^{m} \rightarrow \mathbb{R}\right)$ is thus large.

Let now $[x]$ be the integer part of a real number $x$ and denote by $\mathcal{W}$ the standard one-dimensional Wiener process on $[0,1]$. We will show the following result, giving conditions for a "classical" behavior of the random walk :

\section{Theorem 1.2}

Let $\Omega=\mathbb{T}^{m}$, for some $m \geq 1$, be endowed with an ergodic translation $T: x \longmapsto x+\alpha$ and Lebesgue measure $\mu$. Denote by $c$ the average constant speed in the Law of Large Numbers.

i) If the $\left(p_{z}\right)_{z \in \Lambda}$ are continuous on $\mathbb{T}^{m}$, then $c \neq 0$ whenever the walk is transient $\mu-$ ae.

ii) If the $\left(p_{z}\right)_{z \in \Lambda}$ are $C_{\text {dir }}^{r, s}\left(\mathbb{T}^{m} \rightarrow \mathbb{R}\right)$ with $r+s>\eta(\alpha)$, then there is (in any asymptotic regime) a constant $\sigma>0$ such that the following quenched CLT holds in its functional form:

$$
\sigma^{-1} n^{-1 / 2}\left(\xi_{[n t]}^{\omega}-c[n t]\right)_{t \in[0,1]} \rightarrow \mathcal{W} \text {, as } n \longrightarrow+\infty \text { under } \mathcal{P}_{0}^{\omega}, \mu-a e .
$$

In the second item of the theorem, the condition $r+s>\eta(\alpha)$ is used for solving coboundary equations via the following classical ingredient :

\section{Lemma 1.3}

Let $r+s>\eta(\alpha)$. If $f$ is $C_{\text {dir }}^{r, s}\left(\mathbb{T}^{m} \rightarrow \mathbb{R}\right)$ with $\int f d \mu=0$, then there exists a continuous function $g: \mathbb{T}^{m} \rightarrow \mathbb{R}$ such that $f=g-T g$.

Ideas in this lemma are due to Arnold [3] and go back to KAM Theory. When $m=1$, this version is lemma 5.1 in [7]. The proof for a general $m \geq 1$ requires a slight modification and is given in the last section for the convenience of the reader. One may notice that the condition of the lemma is in some sense "optimal" already when $m=1$, since a Functional Analysis result due to Meyer states that there always exists a $C^{1}\left(\mathbb{T}^{1} \rightarrow \mathbb{R}\right)$ map $f$ with $\int f d \mu=0$ such that $f$ cannot be written $f=g-g \circ T$ for any continuous $g: \mathbb{T}^{1} \rightarrow \mathbb{R}$ (see Herman [15], p 187). It is interesting to observe that the condition appearing in theorem $1.2 \mathrm{ii}$ ) is the same as that of lemma 1.3. In view of the characterization of the CLT in the recurrent case recalled in the presentation when $L=R=1$, this is an indication that theorem 1.2 is sharp in the recurrent case. For instance, assuming that $L=R=1, m=1$ and $\eta(\alpha)=1$, one may take for $\log \left(p_{-1} / p_{1}\right)$ any $C^{1, \delta}\left(\mathbb{T}^{1} \rightarrow \mathbb{R}\right)$ function (with $\delta>0)$ with zero-mean and this gives a recurrent environment where the CLT holds. In the same context, we also indicate the existence of an example in [10] of a continuous $\log \left(p_{-1} / p_{1}\right)$ on $\mathbb{T}^{1}$ with zero mean such that the random walk has a Sinaï type behavior (with a different scaling).

In the transient cases, as mentionned above, it is probable that the CLT already holds in the same context under a continuity hypothesis, but with a random centering. Since a typical centering in [13] is given by the ergodic sum of a quite general function, the condition $r+s>\eta(\alpha)$ appears very natural in order to ensure that the centering is not random. 
Plan of the article. In section 2, we recall definitions and the relevant results from [8, 9]. In section 3, we detail the Harmonic Coordinates formalism introduced by Kozlov in [18] for proving a CLT and provide sufficient conditions for it to be satisfied. In the last section, we show regularity results and then establish theorem 1.2.

Conventions. Implicitly in the whole text we suppose that $\min \{L, R\} \geq 2$. The situation $\min \{L, R\}=1$ requires direct adaptations. We omit the dependence in $\omega$, except when stating results. For a random variable $f$ on $(\Omega, \mathcal{F})$, we write $T f$ for $f \circ T$ and next often simplify $T^{k} f$ into $f(k)$, for $k \in \mathbb{Z}$. We finally set, as in the presentation, $d=L+R-1$.

\section{Definitions and former results}

We present the random matrix $M$ with which results are expressed. We first introduce exit probabilities of finite intervals.

\subsection{Exit probabilities, matrix $M$}

\section{Definition 2.1}

i) Let integers $a<b$. For $k \in[a-L+1, b+R-1]$, set :

$$
\left\{\begin{array}{l}
P_{k}(a, b, \pm)=\mathcal{P}_{k}^{\omega}\{\text { leave }] a+1, b-1[\text { by the right/left side }\} \\
P_{k}(a, b, \zeta)=\mathcal{P}_{k}^{\omega}\{\text { leave }] a+1, b-1[\text { at } \zeta\}, \text { for } \zeta \in\{a-l\}_{0 \leq l \leq L-1} \cup\{b+r\}_{0 \leq r \leq R-1} .
\end{array}\right.
$$

ii) Let integers $a<b$. For $k \in[a-L+1, b+R-1]$ and $\zeta \in\{a-l\}_{0 \leq l \leq L-1} \cup\{b+r\}_{0 \leq r \leq R-1} \cup\{ \pm\}$, define the discrete gradient:

$$
V_{k}(a, b, \zeta)=\left(g_{k+R-i}(a, b, \zeta)\right)_{1 \leq i \leq d} \in \mathbb{R}^{d} \text {, where } g_{k}(a, b, \zeta)=P_{k}(a, b, \zeta)-P_{k+1}(a, b, \zeta)
$$

iii) Let integers $a<b$ and $k \in[a-L+1, b+R-1]$. Define a global right-gradient and a global left-gradient respectively by:

$$
\left\{\begin{array}{l}
\mathcal{R}_{k}(a, b)=\wedge_{j=1}^{R} V_{k}(a, b, b+R-j) \\
\mathcal{L}_{k}(a, b)=\wedge_{j=1}^{L} V_{k}(a, b, a+1-j) .
\end{array}\right.
$$

The definitions are naturally extended to half-infinite intervals, when their meaning is clear. About wedge-products, one may for instance consult Federer [12], chapter one.

For matrix products purposes, we now introduce cocycles notations :

$$
A_{n}=\left\{\begin{array}{cc}
T^{n-1} A \cdots T A A, & n \geq 1 \\
I, & n=0 \\
T^{-n} A^{-1} \cdots T^{-1} A^{-1}, & n \leq-1
\end{array}\right.
$$

The matrix $M$ arises as a circulation matrix when analyzing the Dirichlet problem in a finite interval $[a, b]$, see lemma 2.5 in [8]. All spaces $\mathbb{R}^{l}$ or $\wedge^{n} \mathbb{R}^{l}$ appearing in the sequel are endowed with their canonical Euclidean structure. 


\section{Definition 2.2}

i) Let $M \in G L_{d}(\mathbb{R})$ be the random matrix :

$$
M=\left(\begin{array}{cccccc}
-a_{1} & \cdots & -a_{R-1} & b_{L} & \cdots & b_{1} \\
1 & 0 & \cdots & \cdots & \cdots & 0 \\
0 & \ddots & 0 & \cdots & \cdots & 0 \\
\vdots & \vdots & \vdots & \vdots & \vdots & \vdots \\
\vdots & \vdots & \vdots & \vdots & \vdots & \vdots \\
0 & \cdots & \cdots & \cdots & 1 & 0
\end{array}\right),
$$

where $M_{i, j}=1_{i=j+1}$ for $2 \leq i \leq d$ and:

$$
M_{1, j}=\left\{\begin{array}{cc}
a_{j}=\left(\frac{p_{R-j}+\cdots+p_{R}}{p_{R}}\right), & 1 \leq j \leq R-1 \\
b_{L+R-j}=\left(\frac{p_{R-1-j}+\cdots+p_{-L}}{p_{R}}\right), & R \leq j \leq d .
\end{array}\right.
$$

ii) The Lyapunov exponents $\gamma_{1}(M, T) \geq \cdots \geq \gamma_{d}(M, T)$ of $M$ with respect to $T$ can be recursively defined by the equalities, for $1 \leq i \leq d$ :

$$
\gamma_{1}(M, T)+\cdots+\gamma_{i}(M, T)=\lim _{n \rightarrow+\infty} \frac{1}{n} \int \log \left\|\wedge^{i} M_{n}\right\| d \mu .
$$

About the Lyapunov spectrum and Oseledec's Theorem [21], see Arnold [2], Ledrappier [19] or Raugi [23]. Observe that $M$ and $M^{-1}$ are bounded, as a consequence of condition (1), so that the limits in (3) are finite. We speak below of the Lyapunov spectrum of $(M, T)$.

When it has sense, we suppose defined in the same way as for $(M, T)$ the Lyapunov spectrum of $\left(A, T^{\delta}\right)$ for a matrix $A$ and any $\delta= \pm 1$.

Similarly, the Lyapunov exponent of a vector $V$ with respect to $(A, T)$ is defined as :

$$
\gamma(V, A, T)=\limsup _{n \rightarrow+\infty} \frac{1}{n} \log \left\|A_{n} V\right\| .
$$

In an invertible context, Oseledec's Theorem also furnishes bases of $\mathbb{R}^{d}$ of the following form :

Theorem 2.3 (See [19])

i) There exists a measurable basis $\left(V_{i}\right)_{1 \leq i \leq d}$ of $\mathbb{R}^{d}$ such that $\left\|V_{i}\right\|=1$ and satisfying :

$$
\lim _{n \rightarrow \pm \infty} \frac{1}{|n|} \log \left\|M_{n} V_{i}\right\|= \pm \gamma_{i}(M, T), \forall 1 \leq i \leq d .
$$

ii) There exists a measurable basis $\left(W_{i}\right)_{1 \leq i \leq d}$ in $\mathbb{R}^{d}$ such that $\left\|W_{i}\right\|=1$ and satisfying :

$$
\lim _{n \rightarrow \pm \infty} \frac{1}{|n|} \log \left\|\left({ }^{t} M^{-1}\right)_{-n} W_{i}\right\|= \pm \gamma_{i}(M, T), \forall 1 \leq i \leq d .
$$

For the whole paper, we fix such bases $\left(V_{i}\right)$ and $\left(W_{i}\right)$, except for the central vectors $V_{R}$ and $W_{R}$, which will now be defined very precisely. Let us first set :

Proposition $2.4($ See $[8])$

i) The exponent $\gamma_{1}\left(\wedge^{R} M, T\right)$ is simple. Let $\mathcal{V}_{R} \in \wedge^{R} \mathbb{R}^{d}$ and $\alpha_{R} \in \mathbb{R}_{+}$be defined by :

$$
\mathcal{V}_{R}=\lim _{n \rightarrow+\infty} \frac{\mathcal{R}_{-1}(-n, 0)}{P_{-1}(-n, 0,-)} \text { and } \alpha_{R}=\frac{1}{P_{0}(-\infty, 1, R)} \lim _{n \rightarrow+\infty} \frac{P_{0}(-n, 1,-)}{P_{-1}(-n, 0,-)} .
$$

Then $(-1)^{R-1} \wedge^{R} M \mathcal{V}_{R}=\alpha_{R} T \mathcal{V}_{R}$ and $\mathcal{V}_{R}$ has maximal Lyapunov exponent for $\left(\wedge^{R} M, T\right)$. 
ii) The exponent $\gamma_{1}\left(\wedge^{L} M^{-1}, T^{-1}\right)$ is simple. Let $\mathcal{V}_{L} \in \wedge^{L} \mathbb{R}^{d}$ and $\alpha_{L} \in \mathbb{R}_{+}$be defined by :

$$
\mathcal{V}_{L}=\lim _{n \rightarrow+\infty} \frac{\mathcal{L}_{-1}(-1, n)}{P_{0}(-1, n,+)}, \alpha_{L}=\frac{1}{P_{0}(-1,+\infty,-L)} \lim _{n \rightarrow+\infty} \frac{P_{0}(-1, n,+)}{P_{1}(0, n,+)} .
$$

Then $(-1)^{L-1} \wedge^{L} M^{-1} T \mathcal{V}_{L}=\alpha_{L} \mathcal{V}_{L}$ and $T \mathcal{V}_{L}$ has maximal Lyapunov exponent for $\left(\wedge^{L} M^{-1}, T^{-1}\right)$.

iii) Let random vectors $\mathcal{W}_{R} \in \wedge^{R} \mathbb{R}^{d}$ with $\left\|\mathcal{W}_{R}\right\|=1, \mathcal{W}_{L} \in \wedge^{L} \mathbb{R}^{d}$ with $\left\|\mathcal{W}_{L}\right\|=1$ and random scalars $\beta_{R}>0, \beta_{L}>0$ be such that:

$$
\left\{\begin{array}{c}
(-1)^{R-1} \wedge^{R}\left({ }^{t} M\right) T \mathcal{W}_{R}=\beta_{R} \mathcal{W}_{R} \\
(-1)^{L-1} \wedge^{L}\left({ }^{t} M\right)^{-1} \mathcal{W}_{L}=\beta_{L} T \mathcal{W}_{L}
\end{array}\right.
$$

and $\mathcal{W}_{R}$ and $\mathcal{W}_{L}$ have maximal exponent for $\left(\wedge^{R}\left({ }^{t} M\right), T^{-1}\right)$ and $\left(\wedge^{L}\left({ }^{t} M\right)^{-1}, T\right)$ respectively.

From the simplicity of $\gamma_{1}\left(\wedge^{R} M, T\right)$ and $\gamma_{1}\left(\wedge^{L} M^{-1}, T^{-1}\right)$ it is classical (cf proposition 2.6 in [9]) to infer that $\gamma_{R}(M, T)$ is simple and that $V_{R}$ and $W_{R}$ are uniquely determined in direction.

To define these vectors more precisely, for instance $V_{R}$ via $\mathcal{V}_{R}$ and $\mathcal{V}_{L}$, we recall a few definitions introduced in [9]. If $x \in \wedge^{n} \mathbb{R}^{d}$ is a non-zero decomposable $n$-vector, we write $S(x)$ for the corresponding $n$-dimensional subspace of $\mathbb{R}^{d}$. Although the precise definitions will not be used, we require the linear maps $\operatorname{Ort}_{n}: \wedge^{n} \mathbb{R}^{d} \rightarrow \wedge^{d-n} \mathbb{R}^{d}, x \longmapsto x^{\perp *}$ introduced in definition 2.3 in $[9]$ and such that $S\left(x^{\perp *}\right)=S(x)^{\perp}$ when $x$ is a non-zero decomposable $n$-vector.

Recall also the bilinear map Int $: \wedge^{R} \mathbb{R}^{d} \times \wedge^{L} \mathbb{R}^{d} \rightarrow \mathbb{R}^{d}$, giving a vector spanning $S(x) \cap S(y)$ if $x$ and $y$ are respectively a non-zero decomposable $R$-vector and a non-zero decomposable $L$-vector such that $S(x) \cap S(y)$ is one-dimensional. Only the bilinear character of Int will be used. Then :

Proposition 2.5 (cf [9])

i) Set :

$$
V_{R}=\frac{\operatorname{Int}\left(\mathcal{V}_{R}, \mathcal{V}_{L}\right)}{\left\|\operatorname{Int}\left(\mathcal{V}_{R}, \mathcal{V}_{L}\right)\right\|} \text { and } \lambda_{R}=\frac{p_{R} \alpha_{R}\left\|\operatorname{Int}\left(T \mathcal{V}_{R}, T \mathcal{V}_{L}\right)\right\|}{p_{-L} \alpha_{L}\left\|\operatorname{Int}\left(\mathcal{V}_{R}, \mathcal{V}_{L}\right)\right\|}
$$

Then $M V_{R}=\lambda_{R} T V_{R}$ and $\gamma\left(V_{R}, M, T\right)=-\gamma\left(V_{R}, T^{-1} M^{-1}, T^{-1}\right)=\gamma_{R}(M, T)$. Up to a nonzero multiplicative constant, $V_{R}$ is the only vector with this property. Also $\log \lambda_{R}$ is bounded and $\int \log \lambda_{R} d \mu=\gamma_{R}(M, T)$.

ii) Set :

$$
W_{R}=\frac{\operatorname{Int}\left(\mathcal{W}_{R}, \mathcal{W}_{L}\right)}{\left\|\operatorname{Int}\left(\mathcal{W}_{R}, \mathcal{W}_{L}\right)\right\|} \text { and } \rho_{R}=\frac{p_{R} \beta_{R}\left\|\operatorname{Int}\left(\mathcal{W}_{R}, \mathcal{W}_{L}\right)\right\|}{p_{-L} \beta_{L}\left\|\operatorname{Int}\left(T \mathcal{W}_{R}, T \mathcal{W}_{L}\right)\right\|}
$$

Then ${ }^{t} M T W_{R}=\rho_{R} W_{R}$ and $\gamma\left(W_{R}, T^{-1}\left({ }^{t} M\right), T^{-1}\right)=-\gamma\left(W_{R},{ }^{t} M^{-1}, T\right)=\gamma_{R}(M, T)$. Up to a non-zero multiplicative constant, $W_{R}$ is the only vector with this property. Also $\log \rho_{R}$ is bounded and $\int \log \rho_{R} d \mu=\gamma_{R}(M, T)$.

iii) We have $S\left(\mathcal{V}_{R}\right)^{\perp}=\operatorname{Vect}\left(W_{R+1}, \cdots, W_{d}\right), S\left(\mathcal{V}_{L}\right)^{\perp}=\operatorname{Vect}\left(W_{1}, \cdots, W_{R-1}\right)$ and similarly $S\left(\mathcal{W}_{R}\right)^{\perp}=\operatorname{Vect}\left(V_{R+1}, \cdots, V_{d}\right), S\left(\mathcal{W}_{L}\right)^{\perp}=\operatorname{Vect}\left(V_{1}, \cdots, V_{R-1}\right)$.

This completes the definition of $M$, of its Lyapunov spectrum and related left and right "eigenvectors". See the introduction of [9] for an explanation of the particularity of $\gamma_{R}(M, T)$ and the specific roles attributed to $V_{R}$ and $W_{R}$. 


\subsection{Recurrence criterion, LLN and invariant measure}

A recurrence criterion for the random walk is recalled below, as well as results on the Law of Large Numbers and a criterion for a non-vanishing speed.

Theorem $2.6(C f[8,9])$

i) If $\gamma_{R}(M, T)=0$, then $\liminf \xi_{n}^{\omega}=-\infty<+\infty=\lim \sup \xi_{n}^{\omega}$ and $n^{-1} \xi_{n}^{\omega} \rightarrow 0, \mathcal{P}_{0}^{\omega}-a e, \mu-a e$.

ii) If $\gamma_{R}(M, T)<0$, then $\xi_{n}^{\omega} \rightarrow+\infty$ and $n^{-1} \xi_{n}^{\omega} \rightarrow c, \mathcal{P}_{0}^{\omega}-a e, \mu-a e$, for some constant $c \geq 0$. Also $c>0$ if and only if:

$$
\left\|\sum_{n \geq 0} \lambda_{R} \cdots T^{n-1} \lambda_{R} T^{n} V_{R}\right\| \in L^{1}(\mu)
$$

iii) If $\gamma_{R}(M, T)>0$, then $\xi_{n}^{\omega} \rightarrow-\infty$ and $n^{-1} \xi_{n}^{\omega} \rightarrow c, \mathcal{P}_{0}^{\omega}-a e, \mu-a e$, for some constant $c \leq 0$. Also $c<0$ if and only if :

$$
\left\|\sum_{n \geq 1}\left(T^{-1} \lambda_{R} \cdots T^{-n} \lambda_{R}\right)^{-1} T^{-n} V_{R}\right\| \in L^{1}(\mu) .
$$

The efficiency of the recurrence criterion and the form of the condition for a non-zero speed, in particular the quite subtle geometrical properties of $V_{R}$, are discussed in [9].

An important tool in the analysis of the model is the sequence of the environments seen from the particle. More precisely, introduce $\left(\omega_{n}\right)_{n \geq 0}$ with $\omega_{n}=T^{\xi_{n}^{\omega}} \omega$. For fixed $\omega$, this sequence is a Markov chain on $\Omega$ with initial point $\omega$ and transition operator :

$$
P f(\omega)=\sum_{z \in \Lambda} p_{z}(\omega) T^{z} f(\omega)
$$

Of fundamental importance, for instance for showing the non-zero-speed criterion, is the existence of a $P$-invariant probability measure equivalent to $\mu$. Kozlov [18] proved that a $P$-invariant probability measure which is absolutely continuous is in fact equivalent. Moreover it is unique. We then state :

\section{Definition 2.7}

Call (IM) the existence of a measurable $\pi \geq 0$ such that $\int \pi d \mu=1$ and $P^{*} \pi=\pi, \mu-$ ae.

The following characterization of $(I M)$ was given in [9]

Theorem 2.8 (See [9])

i) If $\gamma_{R}(M, T)=0$, then: $(I M) \Leftrightarrow \exists \varphi \in L^{1}(\mu), \varphi>0, \mu-a . s$, with $\lambda_{R}=\varphi / T \varphi$.

ii) If $\gamma_{R}(M, T)<0$, then : $(I M) \Leftrightarrow\left\|\sum_{n \geq 0} \lambda_{R} \cdots T^{n-1} \lambda_{R} T^{n} V_{R}\right\| \in L^{1}(\mu)$.

iii) If $\gamma_{R}(M, T)>0$, then: $(I M) \Leftrightarrow\left\|\sum_{n \geq 1}\left(T^{-1} \lambda_{R} \cdots T^{-n} \lambda_{R}\right)^{-1} T^{-n} V_{R}\right\| \in L^{1}(\mu)$.

For the sequel, notice that :

$$
P^{*} f(\omega)=\sum_{z \in \Lambda} T^{-z} p_{z}(\omega) T^{-z} f(\omega) .
$$

The next step, as developed in [18], is now concerned with the Central Limit Theorem. 


\section{The Harmonic Coordinates formalism for the CLT}

We describe a general strategy for proving the validity of the functional Central Limit Theorem. As in [7], we use the notion of Harmonic coordinates introduced by Kozlov in [18]. We keep the general setting defined in the introduction. The environment is particularized to the Torus only in the next section.

\section{Definition 3.1}

Given $\pi \in L^{1}(\mu)$ realizing $(I M)$, consider $L_{\pi}^{2}(\Omega \times \Lambda)$ endowed with the following norm :

$$
\|f\|_{L_{\pi}^{2}(\Omega \times \Lambda)}=\left(\sum_{z \in \Lambda} \int|f(\omega, z)|^{2} p_{z}(\omega) \pi(\omega) d \mu(\omega)\right)^{1 / 2} .
$$

For a function $f: \Omega \times \Lambda \rightarrow \mathbb{R}$, set $\tilde{P} f(\omega, z)=\sum_{z^{\prime} \in \Lambda} p_{z^{\prime}}\left(T^{z} \omega\right) f\left(T^{z} \omega, z^{\prime}\right)$. Introduce also the harmonic set $H=\left\{f \in L_{\pi}^{2}(\Omega \times \Lambda) \mid \tilde{P} f=0, \mu-a e\right\}$.

Remark. - As detailed in [18], $\tilde{P}$ is the transition operator of the enlarged Markov chain $\left(\omega_{n}, z_{n}\right)_{n \geq 0}$ on $\Omega \times \Lambda$, where formally $z_{n}$ is the $(n+1)$-step $z_{n}=\xi_{n+1}^{\omega}-\xi_{n}^{\omega}$.

\section{Definition 3.2}

Call "Harmonic Coordinates" and write $(H C)$ the existence of $\pi \in L^{1}(\mu)$ realizing $(I M)$ and $x(\omega, z) \in H$ and $u \in L^{1}(\mu)$ with $\int u d \mu=0$ such that :

$$
\forall z \in \Lambda, z=x(\omega, z)+c+U(\omega, z),
$$

where $c$ is the average speed of the random walk and $U(\omega, z)$ is the $(u, T)$-cocycle :

$$
U(\omega, z)=\left\{\begin{array}{cc}
\sum_{n=0}^{z-1} u\left(T^{n} \omega\right), & z \geq 1 \\
0, & z=0 \\
-\sum_{n=z}^{-1} u\left(T^{n} \omega\right), & z \leq-1
\end{array}\right.
$$

If $(H C)$ is verified, set $\sigma=\|x\|_{L_{\pi}^{2}(\Omega \times \Lambda)}$. In this case $\sigma>0$ (otherwise integration of (5) with respect to $\mu$ gives $z=c$ for $z \in \Lambda$, which is impossible).

The following result is due to Kozlov [18] (see also [10] for all details ; the proof in [18] is valid in the recurrent case, but complicated and in fact does not work in the transient cases). Denote by $\mathcal{W}$ the standard Wiener process on $[0,1]$ and by $[a]$ the integer part of a real number $a$.

Theorem 3.3 (Kozlov [18], theorem 3)

Assume that $(H C)$ holds with the decomposition (5). If $\gamma_{R}(M, T) \neq 0$, suppose furthermore that $u=g-T g$ with $g \in L^{\infty}(\mu)$. Then the following quenched functional convergence is satisfied :

$$
\sigma^{-1} n^{-1 / 2}\left(\xi_{[n t]}^{\omega}-c[n t]\right)_{t \in[0,1]} \rightarrow \mathcal{W}, \text { as } n \longrightarrow+\infty, \quad \text { under } \mathcal{P}_{0}^{\omega}, \mu-a e
$$

We now reformulate condition $(H C)$ using the matrix $M$. The strategy is to express $x(\omega, z)$ in terms of $u$ and then to find $u$ via the equation $\tilde{P} x=0$. In the sequel $\left(e_{i}\right)_{1 \leq i \leq d}$ denotes the canonical basis of $\mathbb{R}^{d}$.

Proposition 3.4

Let $\pi \in L^{1}(\mu)$ realize $(I M)$. Then $(H C)$ holds if and only if there exists $Y \in \mathbb{R}^{d}$ such that :

$$
Y=M T^{-1} Y+\frac{c}{p_{R}} e_{1}
$$

with the properties $\left\langle Y, e_{1}\right\rangle \in L^{1}(\mu), \int\left\langle Y, e_{1}\right\rangle d \mu=1$ and $\int\left\langle Y, e_{1}\right\rangle^{2} \pi d \mu<+\infty$. In this case, the random variable $u$ appearing in $(H C)$ is given by $u=1-T^{1-R}\left\langle Y, e_{1}\right\rangle$. 
Proof of the proposition :

Notice first that the relation $\tilde{P} x=0$ can be rewritten in the form $\sum_{z \in \Lambda} p_{z}(\omega) x(\omega, z)=0$. Using (5), the latter is the equality:

$$
\sum_{z=-L}^{+R} p_{z}(\omega)(z-U(\omega, z)-c)=0
$$

or equivalently :

$$
\sum_{r=1}^{R} p_{r}\left(r-\left(u+\cdots+T^{r-1} u\right)\right)-\sum_{l=1}^{L} p_{-l}\left(l-\left(T^{-1} u+\cdots+T^{-l} u\right)\right)=c .
$$

Setting $y=1-u$, an Abel transform then furnishes :

$$
\sum_{r=0}^{R-1} T^{r} y\left(p_{R}+\cdots+p_{r+1}\right)-\sum_{l=1}^{L} T^{-l} y\left(p_{-l}+\cdots+p_{-L}\right)=c .
$$

Introduce now $Y={ }^{t}\left(T^{R-1} y, \cdots, y, \cdots, T^{-L+1} y\right)$. The condition $\tilde{P} x=0$ is thus equivalent to $Y=M T^{-1} Y+\frac{c}{p_{R}} e_{1}$.

The second and third conditions in the statement of the proposition are obvious. Finally, observe that the requirement $x \in L_{\pi}^{2}(\Omega \times \Lambda)$ is equivalent to $\int T^{l} y^{2} \pi d \mu<+\infty$, for any fixed $l \in \mathbb{Z}$. Indeed, $\Lambda$ is finite and for $z \in \Lambda$ the equation $\pi=P^{*} \pi$ (see (4)) provides $T^{-z} \pi \leq \pi / \varepsilon$, where $\varepsilon>0$ is defined in condition (1). Thus for any $l \in \mathbb{Z}$, we have $C_{l}^{-1} \pi \leq T^{l} \pi \leq C_{l} \pi$, for some constant $C_{l}>0$. This concludes the proof of the proposition.

We now distinguish between recurrent and transient cases.

\section{Proposition 3.5}

If $\gamma_{R}(M, T)=0$, then $(H C)$ is equivalent to the existence of some measurable $\varphi>0$ with $\varphi$ and $1 / \varphi$ simultaneously in $L^{1}(\mu)$ such that $\lambda_{R}=\varphi / T \varphi$.

Proof of the proposition:

Recall first that in the recurrent case the average speed is $c=0$. Assume now that $(H C)$ is satisfied. From proposition 3.4, there exists a non-zero $Y$ with a first coordinate (and in fact $\|Y\|$ ) in $L^{1}(\mu)$ such that $Y=M T^{-1} Y$. As a result, the Lyapunov exponents $\gamma\left(T^{-1} Y, M, T\right)$ and $\gamma\left(Y, M^{-1}, T^{-1}\right)$ are equal to zero. However, this property is only verified for vectors colinear to $V_{R}$, so there exists $\gamma$ such that $Y=\gamma T V_{R}$.

Therefore $\gamma \in L^{1}(\mu)$. Next, $\{\gamma \neq 0\}$ is clearly $T$-invariant. As $\mu\{\gamma \neq 0\}>0$, we get $\mu\{\gamma \neq 0\}=1$, by ergodicity. Then $\lambda_{R}=\gamma / T^{-1} \gamma, \mu-a e$. On the other hand, theorem 2.8 gives $\varphi>0$ in $L^{1}(\mu)$ such that $\lambda_{R}=\varphi / T \varphi$. Thus $\gamma T \varphi$ is $T$-invariant and consequently equal to a non-zero constant. This ends the first direction of the proof.

Reciprocally, let $\lambda_{R}=\varphi / T \varphi$ with some $\varphi>0$ be such that $\varphi$ and $1 / \varphi$ are in $L^{1}(\mu)$. Then $(I M)$ is verified, by theorem 2.8. Concerning $(H C)$, set $Y^{\prime}=T\left(V_{R} / \varphi\right)$. We have $M T^{-1} Y^{\prime}=Y^{\prime}$, with $\left\|Y^{\prime}\right\| \in L^{1}(\mu)$ and $1 /\left\|Y^{\prime}\right\| \in L^{1}(\mu)$. From the further lemma 3.6, we have $\int\left\langle Y^{\prime}, e_{1}\right\rangle d \mu \neq 0$ and can therefore choose $Y=Y^{\prime} / \int\left\langle Y^{\prime}, e_{1}\right\rangle d \mu$. It finally remains to check the last condition of proposition 3.4. Observe that it is enough to show that:

$$
\int\left(1 / \varphi^{2}\right) \pi d \mu<+\infty
$$

We prove this last assertion. By proposition 4.1 in [9], we have the description $\pi=x / p_{R}$, where the vector $X={ }^{t}\left(T^{-R+1} x, \cdots, x, \cdots, T^{L-1} x\right)$, up to a non-zero multiplicative constant, checks by proposition 4.3 iii) and relation (34) in [9] : 


$$
X=T^{-R+2 t} \Phi^{-1} T^{-R+2}\left(\frac{W_{R} \varphi}{\left\langle V_{R}, W_{R}\right\rangle}\right),
$$

for some bounded matrix $\Phi$ (cf definition 4.2 of [9]). Since $\left\|W_{R}\right\|=1$ and $\left|\left\langle V_{R}, W_{R}\right\rangle\right| \geq C>0$ for some constant $C$ (cf proposition 3.16 of [9]), we get $\pi \leq C \varphi$ for another constant $C$. As $1 / \varphi$ is integrable, this concludes the proof of $(6)$.

It remains to prove the non-degeneracy claim, used in the proof of the last proposition. It can be reformulated in the following way:

\section{Lemma 3.6}

Let $Y \in \mathbb{R}^{d}$ be such that $\left\langle Y, e_{1}\right\rangle \in L^{1}(\mu)$ and $M Y=T Y$. If $\int\left\langle Y, e_{1}\right\rangle d \mu=0$, then $Y=0, \mu-a e$.

Proof of the lemma:

Set $T^{-1} y=\left\langle Y, e_{1}\right\rangle$. The relation $M Y=T Y$ gives $Y=\left(T^{-i} y\right)_{1 \leq i \leq d}$ and :

$$
y+\sum_{r=1}^{R-1} \frac{p_{R-r}+\cdots+p_{R}}{p_{R}} T^{-r} y-\sum_{l=1}^{L} \frac{p_{-l}+\cdots+p_{-L}}{p_{R}} T^{-R+1-l} y=0
$$

or equivalently :

$$
\sum_{r=1}^{R} p_{r}\left(T^{-R+1} y+\cdots+T^{-R+r} y\right)-\sum_{l=1}^{L} p_{-l}\left(T^{-R+1-l} y+\cdots+T^{-R} y\right)=0 .
$$

Let now $\left(h_{n}\right)_{n \in \mathbb{Z}}$ be the $\left(T^{-R+1} y, T\right)-$ cocycle :

$$
h_{n}=\left\{\begin{array}{cc}
\sum_{n=0}^{n-1} T^{n-R+1} y, & n \geq 1, \\
0, & z=0 \\
-\sum_{n=z}^{-1} T^{n-R+1} y, & n \leq-1 .
\end{array}\right.
$$

From the previous relation, this cocycle is harmonic in the sense that $h_{n}=\sum_{z \in \Lambda}\left(T^{n} p_{z}\right) h_{n+z}$, for $n \in \mathbb{Z}$. Suppose now that the result is not true. Without loss of generality, let then $\omega \in \Omega$ satisfy $h_{1}(\omega)>0$, the Law of Large Numbers $h_{n}(\omega) / n \rightarrow 0$ and the recurrence of $h_{n}(\omega)$ to 0 as $n \rightarrow \pm \infty$ (Atkinson-Kesten's Theorem, see [4]). We shall contradict some "Maximum Principle".

Set $n_{0}=1$ and let $n_{1} \in\left[n_{0}-L, n_{0}+R\right]$ be such that $h_{n_{1}}(\omega)=\max \left\{h_{p}(\omega) \mid p \in \Lambda+n_{0}\right\}$. First of all $h_{n_{1}}(\omega)>h_{n_{0}}(\omega)$, otherwise $h(\omega)$ is constant on the box $\left[n_{0}-L, n_{0}+R\right]$ and this property propagates to $\mathbb{Z}$, contradicting $h_{0}(\omega)=0$. Suppose for instance that among the possible $n_{1}$, some are $>n_{0}$ and take the right-extremalest in $\Lambda+n_{0}$. Proceed now similarly with $n_{1}$ and define $n_{2}$. Then $h_{n_{2}}(\omega)>h_{n_{1}}(\omega)$ and necessarily $n_{2}>n_{1}$, otherwise this contradicts the choice of $n_{1}$. Recursively, one builds an increasing sequence $\left(n_{p}\right)_{p \geq 0}$ such that $h_{n_{p+1}}(\omega)>h_{n_{p}}(\omega)$ and $n_{p}<n_{p+1} \leq n_{p}+R$.

In a second step, we claim that for any $\eta>0$, there exists an infinite increasing sequence $\left(m_{n}\right)_{n \geq 0}$ with $m_{n}<m_{n+1} \leq m_{n}+\max \{L, R\}$ such that $h_{m_{n}} \leq \eta$. Indeed, as $h_{0}(\omega)=0$, when starting from zero and proceeding as above, one builds a sequence $h_{m_{n}} \leq 0$ with $m_{n}$ monotonically tending either to $+\infty$ or to $-\infty$. The first situation is what we wish, so suppose to be in the second case. As $\left(h_{n}\right)$ is recurrent in the future, there are arbitrary large positive $n$ such that $h_{n} \leq \eta$. Using again the previous argument, there either exists a $R$-dense infinite sequence in $[n,+\infty)$ tending to $+\infty$ or a $L$-dense infinite sequence in $(-\infty, n]$ tending to $-\infty$ such that $h_{i} \leq \eta$ on this subsequence. The first case gives the result, but in the second case we get a $L$-dense sequence in $[0, n]$ with the desired property. Iterating the argument, we obtain the claim.

Let now $\eta=h_{n_{0}}(\omega) / 2$. If for some $k$ and $m$ such that $n_{k}<m \leq n_{k}+R$ we have $h_{m} \leq \eta$, then, using that $h_{n_{k}}(\omega)=\sum_{z \in \Lambda} T^{n_{k}} p_{z}(\omega) h_{n_{k}+z}(\omega)$ and $h_{n_{k+1}}(\omega)$ is the maximum of $h_{i}(\omega), i \in n_{k}+\Lambda$ : 


$$
h_{n_{k+1}}(\omega)-h_{n_{k}}(\omega) \geq \frac{T^{n_{k}} p_{m-n_{k}}(\omega)\left(h_{n_{k}}(\omega)-h_{m}(\omega)\right)}{\sum_{z \in \Lambda \backslash\left\{m-n_{k}\right\}} T^{n_{k}} p_{z}(\omega)} \geq \varepsilon\left(h_{n_{0}}(\omega)-\eta\right)=\varepsilon h_{n_{0}}(\omega) / 2 .
$$

As the $\left(n_{p}\right)_{p \geq 0}$ and the $\left(m_{p}\right)_{p \geq 0}$ are $\max \{L, R\}$-dense in some interval $[A,+\infty)$, one easily contradicts the Law of Large Numbers $h_{n}(\omega) / n \rightarrow 0$. Finally, $h_{1}=0, \mu-a e$. Thus $Y=0, \mu-a e$. This concludes the proof of the lemma.

Remark - A direct application of proposition 3.5 is the situation when $H={ }^{t}(1, \cdots, 1) \in \mathbb{R}^{d}$ is such that $M H=H, \mu-a e$. Indeed in this case, $H$ has zero exponent with respect to $(M, T)$ and to $\left(M^{-1}, T^{-1}\right)$ and is then colinear to $V_{R}$. It is direct that $H$ is in fact a constant multiple of $V_{R}$. Therefore $\lambda_{R}=1$ and a non-degenerate quenched CLT holds. However this last property can be checked rather directly, since the condition $M H=H$ can be rewritten as $\sum_{z \in \Lambda} z p_{z}=0$, as in the beginning of the proof of lemma 3.6. The local drift is zero and the random walk is in fact a martingale in each environment.

We now turn to the transient cases.

\section{Proposition 3.7}

i) If $\gamma_{R}(M, T)<0$, then condition $(H C)$ holds in the case when:

$$
\int\left(\sum_{p \geq 1}\left(T^{-1} \lambda_{R} \cdots T^{-p} \lambda_{R}\right)\right)^{2}\left\|\sum_{n \geq 0}\left(\lambda_{R} \cdots T^{n-1} \lambda_{R}\right) T^{n} V_{R}\right\| d \mu<+\infty .
$$

ii) If $\gamma_{R}(M, T)>0$, then condition $(H C)$ holds in the case when:

$$
\int\left(\sum_{p \geq 0}\left(\lambda_{R} \cdots T^{p-1} \lambda_{R}\right)^{-1}\right)^{2}\left\|\sum_{n \geq 1}\left(T^{-1} \lambda_{R} \cdots T^{-n} \lambda_{R}\right)^{-1} T^{-n} V_{R}\right\| d \mu<+\infty .
$$

Proof of the proposition:

We restrict to case $i$ ) since $i i)$ is similar. As $\sum_{n>0}\left(\lambda_{R} T^{-1} \lambda_{R} \cdots T^{-n+1} \lambda_{R}\right)$ is greater than some positive constant, $(I M)$ holds by theorem 2.8. Also, the average speed $c$ of the random walk is $>0$ by theorem 12.6. Consider next the equation $Y=M T^{-1} Y+c e_{1} / p_{R}$ of proposition 3.4. To find $Y$, we decompose it in the form :

$$
Y=H+K+\gamma T V_{R} \text {, where } H \in S\left(T\left(\mathcal{W}_{L}\right)^{\perp *}\right), K \in S\left(T\left(\mathcal{W}_{R}\right)^{\perp *}\right), \gamma \in \mathbb{R} .
$$

Similarly, we decompose $e_{1}$ with respect to the same subspaces : $e_{1}=H_{0}+K_{0}+\gamma_{0} T V_{R}$. By Oseledec's Theorem, the equation $Y=M T^{-1} Y+c e_{1} / p_{R}$ is equivalent to :

$$
H=M T^{-1} H+\frac{c}{p_{R}} H_{0}, K=M T^{-1} K+\frac{c}{p_{R}} K_{0}, \gamma=\lambda_{R} T^{-1} \gamma+\gamma_{0} .
$$

By proposition 3.16 in [9], $H_{0}, K_{0}$ and $\gamma_{0}$ are bounded. Proceeding as in the proof of theorem 1.8 ii) of [9], using for instance the Poincaré recurrence Theorem, it is not hard to show that the solutions of the previous equations are given by :

$$
\left\{\begin{array}{c}
H=-c \sum_{n \geq 1} T^{-1} M^{-1} \cdots T^{-n+1} M^{-1} T^{-n}\left(\frac{H_{0}}{p_{R}}\right) \\
K=c \sum_{n \geq 0} M T^{-1} M \cdots T^{-n+1} M T^{-n}\left(\frac{K_{0}}{p_{R}}\right) \\
\gamma=c \sum_{n \geq 0} T^{-n}\left(\frac{\gamma_{0}}{p_{R}}\right) \lambda_{R} T^{-1} \lambda_{R} \cdots T^{-n+1} \lambda_{R} .
\end{array}\right.
$$


Proposition 3.17 in [9]) next implies that $H$ and $K$ are bounded quantities. Remark also, by definition of $W_{R}$ and proposition $2.5 \mathrm{iii}$ ) that :

$$
\gamma_{0}=\frac{\left\langle e_{1}, T W_{R}\right\rangle}{\left\langle T V_{R}, T W_{R}\right\rangle} .
$$

Theorem 3.4 and proposition 3.16 of [9] imply that $\left|\gamma_{0}\right|$ is bounded away from 0 and $+\infty$.

Next, by corollary 4.4 of [9], the quantity $\left\|\sum_{n>0}\left(\lambda_{R} \cdots T^{n-1} \lambda_{R}\right) T^{n} V_{R}\right\|$ is greater than some positive constant. As a result, $\sum_{n>0}\left(\lambda_{R} T^{-1} \lambda_{R} \cdots \bar{T}^{-n+1} \lambda_{R}\right)$ belongs to $L^{2}(\mu)$, as well as $\left\langle Y, e_{1}\right\rangle$. By proposition 4.3 and relations (35) and (36) of [9] (and also proposition 3.16, giving that $\left|\left\langle V_{R}, W_{R}\right\rangle\right|$ is bounded away from 0 and $\left.+\infty\right)$, for some constant $C$ we have the inequality :

$$
\pi \leq C\left(1+\left\|\sum_{n \geq 0}\left(\lambda_{R} \cdots T^{n-1} \lambda_{R}\right) T^{n} V_{R}\right\|\right) .
$$

The integrability condition then implies that $\int\left\langle Y, e_{1}\right\rangle^{2} \pi d \mu<+\infty$. It therefore remains to check that $\int\left\langle Y, e_{1}\right\rangle d \mu=1$. From propositions 4.1 and 4.3 of [9] (noticing that ${ }^{t} \Phi e_{1}=\Phi e_{1}=e_{1}$, where $\Phi$ is introduced in definition 4.2 of the same reference), there is $X \in L^{1}(\mu)$ such that $\pi=\left\langle X, e_{1}\right\rangle / p_{R}$ and $T^{-1} X={ }^{t} M X+c e_{1}$, where $c$ is still the average speed of the random walk. Using the equation satisfied by $Y$, we get that :

$$
\begin{aligned}
\langle Y, X\rangle & =\frac{c}{p_{R}}\left\langle e_{1}, X\right\rangle+\left\langle T^{-1} Y,{ }^{t} M X\right\rangle \\
& =\frac{c}{p_{R}}\left\langle e_{1}, X\right\rangle+\left\langle T^{-1} Y, T^{-1} X\right\rangle-c\left\langle T^{-1} Y, e_{1}\right\rangle
\end{aligned}
$$

Since $c>0, \int \pi d \mu=1$ and $X, Y$ are in $L^{1}(\mu)$, we obtain $\int\left\langle Y, e_{1}\right\rangle d \mu=1$. This ends the proof of the proposition.

Remark. - In the transient case, the hypotheses of proposition 3.7 are for instance verified under a condition of uniform convergence. As detailed later, such a property holds under an assumption of unique ergodicity together with continuity of the data. By decomposition (5), this is enough to guarantee that $\left(\xi_{n}^{\omega}-n c-U\left(\omega, \xi_{n}^{\omega}\right)\right)$ is a martingale and thus that $\sigma^{-1} n^{-1 / 2}\left(\xi_{n}^{\omega}-n c-U\left(\omega, \xi_{n}^{\omega}\right)\right)$ converges to standard Brownian Motion, $\mu-a e$. However the fluctuations of $U\left(\omega, \xi_{n}^{\omega}\right)$ may be large. In an independent medium, the case when $n^{-1 / 2} U\left(\omega, \xi_{n}^{\omega}\right)$ satisfies a CLT was considered by Zeitouni [28] for the nearest-neighbour model in an annealed setting (cf also theorem 4.3 in [9] for the $(L, 1)$-model). Deducing from the above general result a meaningful property for the random walk, in the sense of showing that $U\left(\omega, \xi_{n}^{\omega}\right)$ can be replaced by a random quantity depending only on the environment, is not easy, cf for instance Goldsheid [13] for the nearest-neighbour model with in fact a different strategy. In the sequel, under a stronger regularity condition and a compatible requirement on the dynamics, we ensure that $u$ is a coboundary. In this situation $U\left(\omega, \xi_{n}^{\omega}\right)$ is bounded and $n c$ can then be taken as centering in the CLT (this is the content of theorem 3.3).

\section{The example of the Torus}

Our aim is to apply propositions 3.5 and 3.7 in a concrete situation. For the rest of the paper, we assume that $\Omega=\mathbb{T}^{m}$, with an ergodic translation $T: x \longmapsto x+\alpha$ and Lebesgue measure $\mu$.

\subsection{Regularity results}

In order to solve additive coboundary equations, we will first prove regularity results. The basic tool will be the following proposition, generalizing the content of section 5.1 in [7]. It is of independent interest. We begin with a lemma on stochastic matrices. 


\section{Lemma 4.1}

Let $S=\left(s_{i, j}\right)_{1 \leq i, j \leq p}$ be a p-square stochastic matrix. Denote by $\left(e_{i}\right)_{1 \leq i \leq p}$ the canonical basis of $\mathbb{R}^{p}$ and let $f_{i}=e_{i}-e_{i+1}, 1 \leq i \leq p-1$. Then :

i) We have : ${ }^{t} S f_{i}=\sum_{1 \leq l \leq p-1}\left(\sum_{l+1 \leq j \leq p} s_{i+1, j}-\sum_{l+1 \leq j \leq p} s_{i, j}\right)$ f $f_{l}$, for $1 \leq i \leq p-1$.

ii) If $\tilde{S}$ is the matrix of ${ }^{t} S$ in restriction to $(1, \cdots, 1)^{\perp}$, then :

$$
\|\tilde{S} x\|_{1} \leq\left(1-p \min \left\{s_{i, j}\right\}\right)\|x\|_{1} \text {, where }\|x\|_{1}=\sum_{1 \leq i \leq p}\left|x_{i}\right| .
$$

Proof of the lemma:

i) We have :

$$
\begin{aligned}
{ }^{t} S f_{i}=\sum_{j=1}^{p}\left(s_{i, j}-s_{i+1, j}\right) e_{j} & =\sum_{1 \leq j \leq p-1}\left(s_{i, j}-s_{i+1, j}\right)\left(f_{j}+\cdots+f_{p-1}+e_{p}\right)+\left(s_{i, p}-s_{i+1, p}\right) e_{p} \\
& =\sum_{1 \leq j \leq p-1}\left(s_{i, j}-s_{i+1, j}\right)\left(f_{j}+\cdots+f_{p-1}\right) \\
& =\sum_{1 \leq l \leq p-1}\left(\sum_{j \leq l} s_{i, j}-\sum_{j \leq l} s_{i+1, j}\right) f_{l}
\end{aligned}
$$

which is a reformulation of the desired expression.

ii) Take $x \in(1, \cdots, 1)^{\perp}$ with $\|x\|_{1}=1$. Let $I_{+}$and $I_{-}$be the set of indices for which respectively $x_{i}>0$ and $x_{i} \leq 0$. Then $\sum_{I_{+}}\left|x_{i}\right|=\sum_{I_{-}}\left|x_{i}\right|=1 / 2$. Using next the fact that $|a-b|=$ $a+b-2 \min (a, b)$, for $a \geq 0$ and $b \geq 0$ :

$$
\begin{aligned}
\sum_{i}\left|\sum_{j} s_{j, i} x_{j}\right| & =\sum_{i}\left|\sum_{j \in I_{+}} s_{j, i}\right| x_{j}\left|-\sum_{j \in I_{-}} s_{j, i}\right| x_{j}|| \\
& \leq \sum_{i}\left(\sum_{j} s_{j, i}\left|x_{j}\right|-2 \min \left\{\sum_{j \in I_{+}} s_{j, i}\left|x_{j}\right|, \sum_{j \in I_{-}} s_{j, i}\left|x_{j}\right|\right\}\right) \\
& \leq 1-p \min \left\{s_{i, j}\right\} .
\end{aligned}
$$

Recall now definition 1.1 on the class $C_{d i r}^{r, s}\left(\mathbb{T}^{m} \rightarrow \mathbb{R}\right)$. We state :

\section{Proposition 4.2}

Let $r \geq 0,0 \leq s \leq 1$ and $A=\left(a_{i j}\right)_{1 \leq i, j \leq p}$ with $C_{\text {dir }}^{r, s}\left(\mathbb{T}^{m} \rightarrow \mathbb{R}\right)$ entries and such that $A^{N}$ has positive entries for some $N \geq 1$. Let $V$ be the unique random vector with positive entries and $\lambda>0$ be the unique random variable satisfying $A V=\lambda T V$ and $\|V\|=1$. Then $\lambda$ and the entries of $V$ are also $C_{d i r}^{r, s}\left(\mathbb{T}^{m} \rightarrow \mathbb{R}\right)$.

Proof of the proposition :

Implicitly $p \geq 2$, otherwise there is nothing to prove. Since $\lambda=\|A V\|>0$, it is enough to concentrate on $V$. Next, we can clearly assume that $N=1$. By continuity and compacity the entries of $A$ then check $1 / C \leq a_{i j} \leq C$ for some constant $C>0$. We still denote by $\left(e_{i}\right)_{1 \leq i \leq p}$ the canonical basis of $\mathbb{R}^{p}$. We define for the proof $V_{0}=e_{1}+\cdots+e_{p}$ and recursively $\left(V_{n}\right)_{n \geq 0}$ by $V_{n+1}=T^{-1} A T^{-1} V_{n}=T^{-1} A \cdots T^{-n-1} A T^{-n-1} V_{0}$.

It is well-known that the direction of $\left(V_{n}\right)$ uniformly converges to that of $V$ at a uniform exponential rate for the Hilbert distance or any analogous distance (see for instance section 1.3 in 
[7] for details). Since $V$ is, uniformly in $\omega \in \mathbb{R} / \mathbb{Z}$, strictly interior to the positive cone of $\mathbb{R}^{p}$, we get that $V_{n} /\left\|V_{n}\right\|$ (or $V_{n} /\left\langle V_{n}, e_{1}\right\rangle$ ) uniformly converges to $V$ (respectively $V /\left\langle V, e_{1}\right\rangle$ ). Thus $V$ is continuous and this gives the result when $r+s=0$. We now examine further regularity. We shall prove the result only when $r \geq 1$, which is the case needed for theorem 1.2 , since the type of an irrational number is $\geq m \geq 1$. We only use uniform convergence arguments and the fact that the differential of $T$ is everywhere equal to the Identity. We fix a direction $e_{q}$ with $1 \leq q \leq m$ and for simplicity write $f^{(k)}$ for $\partial^{k} f / \partial x_{q}^{k}, k \geq 1$.

We first work on the expression $T V_{n+1}=A V_{n}$. Write $V_{n}=\left(v_{i, n}\right)_{1 \leq i \leq p}$ and define $w_{i, n}$ by $w_{1, n}=1$ and $w_{i, n}=v_{i, n} / v_{i-1, n}$, for $2 \leq i \leq p$. Since $T v_{i, n+1}=\sum_{1 \leq j \leq p} a_{i j} v_{j, n}$, we get for $i \geq 2$ :

$$
T w_{i, n+1}=\frac{\sum_{1 \leq j \leq p} a_{i, j} v_{j, n}}{\sum_{1 \leq j \leq p} a_{i-1, j} v_{j, n}}=\frac{\sum_{1 \leq j \leq p} a_{i, j} w_{1, n} \cdots w_{j, n}}{\sum_{1 \leq j \leq p} a_{i-1, j} w_{1, n} \cdots w_{j, n}} .
$$

When computing the logarithmic partial derivative of $T w_{i, n+1}$ in direction $e_{q}$ we obtain :

$$
\begin{aligned}
\frac{T w_{i, n+1}^{\prime}}{T w_{i, n+1}} & =\frac{\sum_{j} a_{i, j}\left(\sum_{l} w_{1, n} \cdots w_{l, n}^{\prime} \cdots w_{j, n}\right)}{\sum_{j} a_{i, j} w_{1, n} \cdots w_{j, n}}-\frac{\sum_{j} a_{i-1, j}\left(\sum_{l} w_{1, n} \cdots w_{l, n}^{\prime} \cdots w_{j, n}\right)}{\sum_{j} a_{i-1, j} w_{1, n} \cdots w_{j, n}} \\
& +\frac{\sum_{j} a_{i, j}^{\prime} w_{1, n} \cdots w_{j, n}}{\sum_{j} a_{i, j} w_{1, n} \cdots w_{j, n}}-\frac{\sum_{j} a_{i-1, j}^{\prime} w_{1, n} \cdots w_{j, n}}{\sum_{j} a_{i-1, j} w_{1, n} \cdots w_{j, n}} .
\end{aligned}
$$

Consequently :

$$
\begin{aligned}
\frac{T w_{i, n+1}^{\prime}}{T w_{i, n+1}} & =\sum_{l} \frac{w_{l, n}^{\prime}}{w_{l, n}}\left[\frac{\sum_{j \geq l} a_{i, j} w_{1, n} \cdots w_{j, n}}{\sum_{j} a_{i, j} w_{1, n} \cdots w_{j, n}}-\frac{\sum_{j \geq l} a_{i-1, j} w_{1, n} \cdots w_{j, n}}{\sum_{j} a_{i-1, j} w_{1, n} \cdots w_{j, n}}\right] \\
& +\left[\frac{\sum_{j} a_{i, j}^{\prime} w_{1, n} \cdots w_{j, n}}{\sum_{j} a_{i, j} w_{1, n} \cdots w_{j, n}}-\frac{\sum_{j} a_{i-1, j}^{\prime} w_{1, n} \cdots w_{j, n}}{\sum_{j} a_{i-1, j} w_{1, n} \cdots w_{j, n}}\right] .
\end{aligned}
$$

Recall that $w_{1, n}^{\prime}=0$, since $w_{1, n}=1$. Set $J_{n}=\left(w_{i+1, n}^{\prime} / w_{i+1, n}\right)_{1 \leq i \leq p-1} \in \mathbb{R}^{p-1}$ and let :

$$
S_{i, j, n}=\frac{a_{i, j} w_{1, n} \cdots w_{j, n}}{\sum_{l} a_{i, l} w_{1, n} \cdots w_{l, n}} \text { and } L_{i, j, n}=\frac{a_{i, j}^{\prime} w_{1, n} \cdots w_{j, n}}{\sum_{l} a_{i, l} w_{1, n} \cdots w_{l, n}} .
$$

The first quantities define a $p$-square stochastic matrix $S_{n}=\left(S_{i, j, n}\right)_{1 \leq i, j \leq p}$. We next introduce a $(p-1)$-square matrice $U_{n}=\left(U_{i, j, n}\right)_{1 \leq i, j \leq p-1}$ and a $(p-1)$-vector $V_{n}=\left(V_{i, n}\right)_{1 \leq i \leq p-1}$ by :

$$
U_{i, j, n}=\sum_{j+1 \leq l \leq p}\left(S_{i+1, l, n}-S_{i, l, n}\right) \text { and } V_{i, n}=\sum_{1 \leq l \leq p}\left(L_{i+1, l, n}-L_{i, l, n}\right) .
$$

By lemma $4.1 i), U_{n}$ is the matrix of ${ }^{t} S_{n}$ in restriction to ${ }^{t}(1 \cdots 1)^{\perp}$ and in the basis $e_{1}-e_{2}, e_{2}-$ $e_{3}, \cdots, e_{p-1}-e_{p}$. Exploiting the fact that the entries of $S_{n}$ are uniformly converging to strictly positive constants, by lemma $4.1 \mathrm{ii}$ ) there is a fixed norm on $\mathbb{R}^{p-1}$ such that the induced norm of $U_{n}$ is less than $1-\delta$, with some fixed $\delta>0$, uniformly in $n$.

Since (9) can be rewritten into $T J_{n+1}=U_{n} J_{n}+V_{n}$, when iterating we deduce :

$$
J_{n}=\sum_{l=0}^{n-1} T^{-1} U_{n-1} \cdots T^{-l} U_{n-l} T^{-l-1} V_{n-l}+T^{-1} U_{n-1} \cdots T^{-n-1} U_{0} T^{-n-1} V_{0}, \quad n \geq 1 .
$$

Since the $V_{n}$ uniformly converge towards some $V$ and in particular remain bounded, we obtain that $J_{n}$ uniformly converges towards $J=\sum_{l \geq 0} T^{-1} U \cdots T^{-l} U T^{-l-1} V$, where $U$ is the uniform limit 
of $\left(U_{n}\right)$. As a result and by definition of $J_{n}$, we get that the $\left(w_{i, n}^{\prime}\right)$ uniformly converge, since the $\left(w_{i, n}\right)$ already uniformly converge. It directly implies that the vector $V_{n} / v_{i, n}$ uniformly converges to a vector $\tilde{V}$ colinear to $V$ with entries admitting continuous partial derivatives in direction $e_{q}$. Since $V=\tilde{V} /\|\tilde{V}\|$, the entries of $V$ also have this property.

Concerning higher regularity, one has for $0 \leq k \leq r-1$ :

$$
J_{n+1}^{(k)}=T^{-1}\left(U_{n} J_{n}^{(k)}\right)+W_{n}, \text { where } W_{n}=T^{-1}\left[\sum_{0 \leq l \leq k-1}\left(\begin{array}{c}
k \\
l
\end{array}\right) U_{n}^{(k-l)} J_{n}^{(l)}+V_{n}^{(k)}\right]
$$

If one inductively supposes that $J_{n}^{(l)}$ uniformly converges for $0 \leq l \leq k-1$, one gets that $J^{(k)}$ uniformly converges in the same way as above, simply replacing $V_{n}$ by $W_{n}$ which is uniformly converging and bounded in $n$. From this, we obtain that $V_{n} / v_{i, n}$ uniformly converges to a vector with $C_{\operatorname{dir}\left(e_{q}\right)}^{k+1}\left(\mathbb{T}^{m} \rightarrow \mathbb{R}\right)$ entries, where $\operatorname{dir}\left(e_{q}\right)$ is for "in direction $e_{q}$ ". Taking now $k=r-1$, one gets for some $s$-Hölder continuous in the $e_{q}$-direction (uniformly on $\mathbb{T}^{m}$ ) $W$ :

$$
T J^{(r-1)}=U J^{(r-1)}+W .
$$

Therefore, as above, $J^{(r-1)}=\sum_{l>0} T^{-1} U \cdots T^{-l} U T^{-l-1} W$. Since $U$ is continuously differentiable in direction $e_{q}$, with a norm less than $1-\delta$, it is clear that for some constant $C$, any $l \geq 1$ and any $\omega_{1}$ and $\omega_{2}$ in $\mathbb{T}^{m}$ one has :

$$
\left\|T^{-1} U \cdots T^{-l} U T^{-l-1} W\left(\omega_{1}\right)-T^{-1} U \cdots T^{-l} U T^{-l-1} W\left(\omega_{2}\right)\right\| \leq C l(1-\delta)^{l} d\left(\omega_{1}, \omega_{2}\right)^{s} .
$$

Therefore $J^{(r-1)}$ is $s$-Hölder in the $e_{q}$-direction, as well as $w_{i}^{(r)}$ and finally $V^{(r)}$. This finally concludes the proof of the proposition.

We shall apply proposition 4.2 , but not directly. Indeed, the matrices $(-1)^{R-1} \wedge^{R} M$ and $(-1)^{L-1} \wedge^{L} M^{-1}$ were shown in [8] to be cone-preserving for some explicit and deterministic cones in $\wedge^{R} \mathbb{R}^{d}$ and $\wedge^{L} \mathbb{R}^{d}$ respectively. Although having a directional contraction property like positive matrices, these matrices do not have non-negative coefficients and, as indicated in [8], are probably not even conjugated to non-negative matrices.

The idea is to study closely the linear action of $(-1)^{R-1} \wedge^{R} M$, for instance, on the edges of a stable cone and to extract a positive matrix via an explicit computation done in [9]. Details are the object of the next proposition.

\section{Proposition 4.3}

Let $r \geq 0,0 \leq s \leq 1$ and assume that the entries of $M$ are $C_{d i r}^{r, s}\left(\mathbb{T}^{m} \rightarrow \mathbb{R}\right)$. Then it is also the case of the entries of $\mathcal{V}_{R}, \mathcal{V}_{L}, V_{R}$ and $\mathcal{W}_{R}, \mathcal{W}_{L}, W_{R}$, as well as $\alpha_{R}, \beta_{R}, \alpha_{L}, \beta_{L}, \lambda_{R}, \rho_{R}$.

Proof of the proposition :

We shall restrict to $\mathcal{V}_{R}, \mathcal{V}_{L}, V_{R}$ and $\alpha_{R}, \alpha_{L}, \lambda_{R}$. We will use proposition 3.11 in [9]. The case of $\mathcal{W}_{R}, \mathcal{W}_{L}, W_{R}$ and $\beta_{R}, \beta_{L}, \rho_{R}$ is treated similarly, using this time proposition 3.7 in [8].

By proposition 2.5, the regularity of $V_{R}$ and $\lambda_{R}$ follows from that of $\mathcal{V}_{R}, \mathcal{V}_{L}, \alpha_{R}$ and $\alpha_{L}$, as the map Int is bilinear and $\operatorname{Int}\left(\mathcal{V}_{R}, \mathcal{V}_{L}\right)$ is always non-zero. By symmetry, we now only consider the case of $\mathcal{V}_{R}$ and $\alpha_{R}$. By proposition 2.4, remark that $\left\langle\mathcal{V}_{R}, e_{1} \wedge \cdots \wedge e_{R}\right\rangle=(-1)^{R}$. We thus get $\alpha_{R}=-\left\langle\wedge^{R} M \mathcal{V}_{R}, e_{1} \wedge \cdots \wedge e_{R}\right\rangle$ and it is then enough to look at the regularity of $\mathcal{V}_{R}$.

We now consider $\mathcal{V}_{R}$ and begin as in the proof of proposition 4.2. We recursively define $\left(Z_{n}\right)$ by $Z_{0}=e_{1} \wedge\left(e_{2}-e_{1}\right) \cdots \wedge\left(e_{R-1}-e_{R-2}\right) \wedge\left(e_{R}-e_{R+1}\right) \in \wedge^{R} \mathbb{R}^{d}$ and next $T Z_{n+1}=(-1)^{R-1} \wedge^{R} M Z_{n}$. Let $\mathcal{C}_{+}$be the polyhedral cone introduced in definition 3.9 of [9], with set of edges $\mathcal{E}_{+}$. As shown 
by proposition 3.11 of the same reference, this cone is stable under the linear action of the class of matrices having "the same form" as $(-1)^{R-1} \wedge^{R} M$ and is minimal for this property. By definition, $Z_{0}$ is in $\mathcal{E}_{+}$and belongs to $\mathcal{C}_{+}$. Therefore $Z_{n}$ belongs to $\mathcal{C}_{+}$for all $n$. We next decompose :

$$
Z_{n}=\sum_{\varphi \in \mathcal{E}_{+}} \alpha_{n}(\varphi) \varphi
$$

One may notice that the $\varphi$ in $\mathcal{E}_{+}$may not form a basis of $\wedge^{R} \mathbb{R}^{d}$, as they are in general much more numerous than the dimension of $\wedge^{R} \mathbb{R}^{d}$. Therefore the way of decomposing $Z_{n}$, even with positive coefficients, may not be unique. We shall choose a particular decomposition, given by the successive application of lemma 3.10 in [9]. More precisely, by this lemma, for $\varphi$ in $\mathcal{E}_{+}$:

$$
(-1)^{R-1} \wedge^{R} M \varphi=\sum_{\psi \in \mathcal{E}_{+}} \beta(\varphi, \psi) \psi,
$$

where for each fixed couple $(\varphi, \psi)$ in $\mathcal{E}_{+}$, the quantity $\beta(\varphi, \psi)$ is non-negative and with the same regularity as the entries of $M$ (with the notations of this lemma, note that the $\alpha_{l, j}$ and $\alpha_{l,-}$ are constants equal to 0 or 1$)$. Since :

$$
(-1)^{R-1} \wedge^{R} M Z_{n}=\sum_{\varphi, \psi \in \mathcal{E}_{+}} \alpha_{n}(\varphi) \beta(\varphi, \psi) \psi,
$$

we recursively define $\left(\alpha_{n}(\varphi)\right)_{\varphi \in \mathcal{E}_{+}}$by :

$$
T \alpha_{n+1}(\varphi)=\sum_{\psi \in \mathcal{E}_{+}} \beta(\psi, \varphi) \alpha_{n}(\psi), \varphi \in \mathcal{E}_{+} .
$$

Consequently $T\left(\alpha_{n+1}(\varphi)\right)_{\varphi \in \mathcal{E}_{+}}=A\left(\alpha_{n}(\varphi)\right)_{\varphi \in \mathcal{E}_{+}}$, where $A=(\beta(\psi, \varphi))_{\varphi, \psi \in \mathcal{E}_{+}}$. One may then observe that for some fixed $s \geq 1$, the matrix $T^{-1} A \cdots T^{-s} A$ (and thus $A^{s}$ ) has strictly positive entries. In fact one may take $s=3 L$ (see the proof of proposition 3.12 in [9]; this is a consequence of the minimality of $\mathcal{C}_{+}$). Applying to $A$ proposition 4.2 , we obtain that for all $\varphi$ and $\psi$ in $\mathcal{E}_{+}$the ratio $\alpha_{n}(\varphi) / \alpha_{n}(\psi)$ converges to a positive map $q(\varphi, \psi)$ which is $C_{d i r}^{r, s}\left(\mathbb{T}^{m} \rightarrow \mathbb{R}\right)$. Fixing $\varphi_{0} \in \mathcal{E}_{+}$, the entries of the $R$-vector :

$$
\tilde{Z}=\frac{\sum_{\varphi \in \mathcal{E}_{+}} q\left(\varphi, \varphi_{0}\right) \varphi}{\sum_{\varphi \in \mathcal{E}_{+}} q\left(\varphi, \varphi_{0}\right)}
$$

are also $C_{d i r}^{r, s}\left(\mathbb{T}^{m} \rightarrow \mathbb{R}\right)$. From the cone contraction property of $(-1)^{R-1} \wedge^{R} M$ (cf proposition 3.11 in [9]) and proposition 4.15 in [8], $\tilde{Z}$ is colinear to $\mathcal{V}_{R}$. Since $\mathcal{V}_{R}=(-1)^{R} \tilde{Z} /\left\langle\tilde{Z}, e_{1} \wedge \cdots \wedge e_{R}\right\rangle$, the entries of $\mathcal{V}_{R}$ are also $C_{d i r}^{r, s}\left(\mathbb{T}^{m} \rightarrow \mathbb{R}\right)$ and this completes the proof of the proposition.

\subsection{Proof of theorem 1.2}

Step 1. We begin with a proof of lemma 1.3. Let $\left(c_{n}(f)\right)_{n \in \mathbb{Z}^{m}}$ be the Fourier expansion of $f$. The equation $f=g-T g$ formally leads to $c_{n}(g)=c_{n}(f) /\left(1-e^{2 i \pi\langle n, \alpha\rangle}\right), n \in \mathbb{Z}^{m}$. Under the hypotheses of the theorem, we show that $\sum_{n \in \mathbb{Z}^{m}}\left|c_{n}(g)\right|<+\infty$, ie $\sum_{n \in \mathbb{Z}^{m}}\left|c_{n}(f)\right| / \operatorname{dist}(\langle n, \alpha\rangle, \mathbb{Z})<+\infty$. Fix $\varepsilon>0$ such that $r+s>\eta+\varepsilon$, where $\eta=\eta(\alpha)$. We denote in the sequel a generic constant $C>0$ depending only $m, r, s, \eta$ and $\varepsilon$. For instance, by definition of the type, if $n \neq 0$ :

$$
\operatorname{dist}(\langle n, \alpha\rangle, \mathbb{Z}) \geq C\|n\|_{\infty}^{-\eta-\varepsilon}
$$

First $\left|c_{n}(f)\right| \leq C\|n\|_{\infty}^{-(r+s)}$ after successive integrations by parts in direction $e_{q}$, where $1 \leq q \leq$ $m$ is chosen so that $\left|n_{q}\right|=\|n\|_{\infty}$ (for the last step, consider the Fourier coefficient of $\partial^{r} f / \partial x_{q}^{r}(x+$ $\left.h e_{q}\right)-\partial^{r} f / \partial x_{q}^{r}(x)$ with a convenient $\left.h\right)$. We shall then prove : 


$$
\sum_{i \geq 1} 2^{i} \sum_{n \in \mathcal{N}_{i}}\|n\|_{\infty}^{-(r+s)}<+\infty
$$

where $\mathcal{N}_{i}:=\left\{n \in \mathbb{Z}^{m} \mid 2^{-i-1} \leq \operatorname{dist}(\langle n, \alpha\rangle, \mathbb{Z})<2^{-i}\right\}$. Next :

$$
\sum_{n \in \mathcal{N}_{i}}\|n\|_{\infty}^{-(r+s)} \leq C \sum_{p \geq p(i)} 2^{-p(r+s)} \operatorname{card}\left\{n \in \mathcal{N}_{i} \mid 2^{p} \leq\|n\|_{\infty}<2^{p+1}\right\},
$$

where $p(i)$ is the first $p$ such that $\left\{n \in \mathcal{N}_{i} \mid 2^{p} \leq\|n\|_{\infty}<2^{p+1}\right\}$ is non-empty. Observe that distinct elements $n$ and $n^{\prime}$ in $\mathcal{N}_{i}$ check $2.2^{-i} \geq \operatorname{dist}\left(\left\langle n-n^{\prime}, \alpha\right\rangle, \mathbb{Z}\right) \geq C\left\|n-n^{\prime}\right\|_{\infty}^{-\eta-\varepsilon}$. Hence $\left\|n-n^{\prime}\right\| \geq$ $C 2^{i / \eta+\varepsilon}$. Comparing volumes, we get $\operatorname{card}\left\{n \in \mathcal{N}_{i} \mid 2^{p} \leq\|n\|_{\infty}<2^{p+1}\right\} \leq C 2^{p m} / 2^{i m /(\eta+\varepsilon)}$. This also implies, by definition of $p(i)$, that $2^{-i} \geq C 2^{-p(i)(\eta+\varepsilon)}$. Since $r+s>m$, we deduce that :

$$
\sum_{n \in \mathcal{N}_{i}}\|n\|_{\infty}^{-(r+s)} \leq C 2^{-p(i)(r+s-m)} 2^{-i m /(\eta+\varepsilon)} .
$$

Therefore $\sum_{n \in \mathcal{N}_{i}}\|n\|_{\infty}^{-(r+s)} \leq C 2^{-i(r+s-m) /(\eta+\varepsilon)} 2^{-i m /(\eta+\varepsilon)}=C 2^{-i(r+s) /(\eta+\varepsilon)}$, which gives (10).

Step 2. We consider the proof of theorem 1.2. Concerning $i)$, if the $\left(p_{z}\right)_{z \in \Lambda}$ are continuous, then $\lambda_{R}$ is continuous too by proposition 4.3. Unique ergodicity of $T$ with respect to Lebesgue measure implies that $n^{-1} \sum_{0 \leq k<n} T^{k} \log \lambda_{R} \rightarrow \gamma_{R}(M, T)$ uniformly on $\mathbb{T}^{m}$. The integrability conditions in the transient cases for a non-zero speed in theorem 2.6 of [9] are then satisfied.

We turn to point $i i)$ and suppose that the $\left(p_{z}\right)_{z \in \Lambda}$ are $C_{d i r}^{r, s}\left(\mathbb{T}^{m} \rightarrow \mathbb{R}\right)$ in the sense of definition 1.1. In the recurrent case $\gamma_{R}(M, T)=0$, since $\lambda_{R}$ and thus $\log \lambda_{R}$ are $C_{d i r}^{r, s}\left(\mathbb{T}^{m} \rightarrow \mathbb{R}\right)$ by proposition 4.3, we get from lemma 1.3 that $\log \lambda_{R}=g-T g$ for some continuous and therefore bounded $g$. One then applies proposition 3.5 and next theorem 3.3 to conclude.

It remains to show the result in the transient cases, which are more delicate. We will suppose for instance that $\gamma_{R}(M, T)<0$. By proposition 3.7, notice first that there exist harmonic coordinates, since $\lambda_{R}$ being continuous, uniformity in the Law of Large Numbers implies the required integrability condition. In order to apply theorem 3.3 and get the result, it remains to show that the map $u$ appearing in the decomposition in harmonic coordinates is $C_{d i r}^{r, s}\left(\mathbb{T}^{m} \rightarrow \mathbb{R}\right)$. In the sequel we shall say "regular" in place of $C_{d i r}^{r, s}\left(\mathbb{T}^{m} \rightarrow \mathbb{R}\right)$. By proposition 3.4, it is equivalent to showing that the entries of the random vector $Y$ solution of $Y=M T^{-1} Y+c e_{1} / p_{R}$ are regular, where $c>0$ is the average speed. As in proposition 3.7 (but not exactly), we decompose :

$$
Y=H+K, \text { where } H \in S\left(T\left(\mathcal{W}_{L}\right)^{\perp *}\right), K \in S\left(T \mathcal{V}_{L}\right) .
$$

Recall that the dimensions of these subspaces are respectively $R-1$ and $L$. Decomposing also according to the same subspaces, let $c e_{1} / p_{R}=H_{0}+K_{0}$. From Oseledec's Theorem, the equation $Y=M T^{-1} Y+c e_{1} / p_{R}$ is equivalent to $H=M T^{-1} H+H_{0}$ together with $K=M T^{-1} K+K_{0}$. Proceeding as in proposition 3.7, $H_{0}$ and $K_{0}$ are bounded and :

$$
\left\{\begin{array}{c}
H=-\sum_{n \geq 1} T^{-1} M^{-1} \cdots T^{-n+1} M^{-1} T^{-n} H_{0} \\
K=\sum_{n \geq 0} M T^{-1} M \cdots T^{-n+1} M T^{-n} K_{0} .
\end{array}\right.
$$

Step 3. We exhibit regular (in the above sense) bases of $S\left(\left(\mathcal{W}_{L}\right)^{\perp *}\right)$ and $S\left(\mathcal{V}_{L}\right)$. It will then result from the Cramer formulas that $H_{0}$ and $K_{0}$ are regular. Mention that the existence of such bases certainly results from general arguments, but it is not hard to construct some explicitly.

First, the $V_{-1}(-1,+\infty,-j)$, for $1 \leq j \leq L$, form a basis of $S\left(\mathcal{V}_{L}\right)$. This is a consequence of the fact that this family is free and lemma 2.4 in [8], as well as $\gamma_{R+1}(M, T)>0$ (see proposition 4.3 in [8]). On the contrary, the family $V_{0}(-\infty, 1, r)$, with $1 \leq r \leq R$, is not free and has rank $R-1$. 
However, for example and similarly, the $V_{0}(-\infty, 1, r), 2 \leq r \leq R$, form a basis of $S\left(\left(\mathcal{W}_{L}\right)^{\perp *}\right)$. We next explicit regular bases. Write :

$$
\mathcal{L}_{-1}(-1,+\infty)=\wedge_{j=1}^{L} V_{-1}(-1,+\infty,-j)=V_{1} \wedge\left(\wedge_{j=2}^{L} V_{j}\right)
$$

where $V_{j}:=\sum_{j \leq l \leq L} V_{-1}(-1,+\infty,-j)$. It is a simple observation that $V_{1}=\left(P_{R-i}(-1,+\infty,+)-\right.$ $\left.P_{R-1-i}(-1,+\infty,+)\right)_{1 \leq i \leq d}$ and that the entries of $\tilde{V}:=V_{1} / P_{0}(-1,+\infty,+)$ with index in $\{R+$ $1, \cdots, d\}$ are zero and the $R$-th entry is 1 . By proposition $2.4 \mathrm{ii}$ ), we have :

$$
\mathcal{V}_{L}=\tilde{V} \wedge\left(\wedge_{j=2}^{L} V_{j}\right)=\tilde{V} \wedge\left(\wedge_{j=2}^{L}\left(V_{j}-\left\langle V_{j}, e_{R}\right\rangle \tilde{V}\right)\right)
$$

Now $\mathcal{V}_{L}$ is regular, by proposition 4.3. The form of the previous vectors then implies that $\tilde{V}$ and all the $V_{j}-\left\langle V_{j}, e_{R}\right\rangle \tilde{V}, 2 \leq j \leq L$ are regular. Indeed, when developing the right hand-side of (12) in the canonical basis of $\wedge^{L} \mathbb{R}^{d}$, the coefficient corresponding to $e_{R+1} \wedge e_{R+2} \cdots \wedge{ }_{j} e_{z} \cdots \wedge e_{d}$ (where $e_{z}$ is at place $j$ ) with $1 \leq z \leq R$ is exactly the $z$-th entry of $V_{j}-\left\langle V_{j}, e_{R}\right\rangle \tilde{V}$. As a result, $B_{1}:=\left\{\tilde{V}, V_{j}-\left\langle V_{j}, e_{R}\right\rangle \tilde{V}, 2 \leq j \leq L\right\}$ is a regular basis of $S\left(\mathcal{V}_{L}\right)$.

Consider now the $V_{0}(-\infty, 1, r), 2 \leq r \leq R$. There is a random $\kappa$ such that :

$$
\mathcal{W}_{L}^{\perp *}=\kappa \wedge_{r=0}^{R-2} V_{0}(-\infty, 1, R-r)=\kappa \wedge_{r=0}^{R-2} V^{r}
$$

where by definition $V^{r}:=V_{0}(-\infty, 1, R)+\cdots+V_{0}(-\infty, 1, R-r)$. From proposition $4.3, \mathcal{W}_{L}$ is regular, as well as its image $\mathcal{W}_{L}^{\perp *}$ under a linear map. Since $\left\langle\wedge_{r=0}^{R-2} V^{r}, e_{1} \wedge \cdots \wedge e_{R-1}\right\rangle=(-1)^{R-1}$, we deduce that $\kappa=(-1)^{R-1}\left\langle\mathcal{W}_{L}^{\perp *}, e_{1} \wedge \cdots \wedge e_{R-1}\right\rangle$ is also regular. Next, as above and due to the form of these vectors, the $V^{r}$, with $0 \leq r \leq R-2$, are regular and thus $B_{2}:=\left\{V^{r}, 0 \leq r \leq R-2\right\}$ is a regular basis of $S\left(\mathcal{W}_{L}^{\perp *}\right)$.

Step 4. We show that the solution $K$ of $K=M T^{-1} K+K_{0}$ is regular. The case of $H$, solution of $H=M T^{-1} H+H_{0}$, is treated in the same way. Let $\tilde{K}$ and $\tilde{K}_{0}$ be the regular components of $K$ and $K_{0}$ respectively in the basis $T B_{1}$. Let $N$ be the $L$-square random matrix giving the coordinates of the images of $B_{1}$ by $M$ in the basis $T B_{1}$. It is clear that $N$ is a regular matrix. We shall show that there are constants $C$ and $0<\delta<1$ such that, for all $n \geq 0$ and all $X \in S\left(\mathcal{V}_{L}\right)$ :

$$
\left\|T^{n} N \cdots T N N X\right\| \leq C \delta^{n}\|X\|
$$

Since :

$$
\tilde{K}=\sum_{n \geq 0} N T^{-1} N \cdots T^{-n+1} N T^{-n} \tilde{K}_{0},
$$

property (13) guarantees that the $k^{t h}$ partial derivative (with $k \leq[r+s]$ ) of the general term $N T^{-1} N \cdots T^{-n+1} N T^{-n} \tilde{K}_{0}$ in any fixed direction is bounded by $\tilde{C} n^{k} \delta^{n}$ for some constant $\tilde{C}$. The result then easily follows by uniform convergence, as in the proof of proposition 4.2 for instance.

It remains to show (13). Recall that $S\left(\mathcal{V}_{L}\right)=S\left(\mathcal{W}_{R}^{\perp *}\right) \oplus \operatorname{Vect}\left(V_{R}\right)$ and that $S\left(\mathcal{V}_{L}\right)=S\left(\mathcal{W}_{R}^{\perp *}\right)$ and $\operatorname{Vect}\left(V_{R}\right)$ are uniformly in a non-singular position, see proposition $\left.3.16 \mathrm{iii}\right)$ in [9]. The result follows from proposition $3.17 i$ ) in [9] and the fact that $T^{n-1} M \cdots T M M V_{R}=T^{n-1} \lambda_{R} \cdots \lambda_{R} T^{n} V_{R}$, where $n^{-1}\left(T^{n-1} \log \lambda_{R}+\cdots+\log \lambda_{R}\right) \rightarrow \gamma_{R}(M, T)<0$, uniformly on $\Omega$ since $\lambda_{R}$ is continuous. This completes the proof of the theorem. 


\section{References}

[1] AliLi, S., Asymptotic behaviour for random walks in random environments, J. Appl. Probab. 36 (1999), no. 2, 334-349.

[2] Arnold, L., Random Dynamical Systems, Springer-Verlag, Berlin, 1998.

[3] Arnold, V., Small denominators. I. Mapping the circle onto itself, Izv. Akad. Nauk SSSR Ser. Mat. 25 (1961), 21-86.

[4] Atkinson, G., Recurrence of co-cycles and random walks, J. London Math. Soc (2), 1976, 13, 486-488.

[5] Bolthausen, E. And Goldsheid, I., Recurrence and transience of random walks in random environments on a strip, Com. Math. Phys., 2000, vol. 214, 429-447.

[6] Bolthausen, E. And Goldsheid, I., Lingering random walks in random environment on a strip, preprint.

[7] Brémont, J., On some random walks on $\mathbb{Z}$ in random medium, Ann. Probab. 30 (2002), no. 3, 1266-1312.

[8] Brémont, J., Random walks on $\mathbb{Z}$ in random medium and Lyapunov spectrum, Annales de l'I.H.P. Prob/Stat, Vol 40, no. 3, 2004, 309-336.

[9] BrÉmont, J., One-dimensional finite range random walk in random medium and invariant measure equation, Annales de l'I.H.P. Prob/Stat, to appear.

[10] BrÉmont, J., Thèse de doctorat, Marches aléatoires en milieu aléatoire sur $\mathbb{Z}$; dynamique d'applications localement contractantes sur le Cercle, Université de Rennes I, 2002.

[11] Conze, J.-P. And Guivarc'H, Y., Marches en milieu aléatoire et mesures quasi-invariantes pour un système dynamique, Colloq. Math. 84/85 (2000), part 2, 457-480. Dedicated to the memory of Anzelm Iwanik.

[12] Federer, H., Geometric measure theory, Springer-Verlag, New York, 1969.

[13] Goldsheid, I., Random walk in random environment : the Central Limit Theorem, to appear in PTRF.

[14] GoldsheID, I., Linear and sublinear growth and the CLT for hitting times of a random walk in random environment on a strip, to appear in PTRF.

[15] Herman, M., Sur la conjugaison différentiable des difféomorphismes du cercle à des rotations, Inst. Hautes Etudes Sci. Publ. Math. No. 49 (1979), 5-233.

[16] KEY, E., Recurrence and transience criteria for a random walk in a random environment, Ann. Probab. 12 (1984), no. 2, 529-560.

[17] Khinchin, A., Continued fractions, The University of Chicago Press, Chicago, Ill.-London 1964 xi+95 pp.

[18] KozLov, S., The averaging method and walks in inhomogeneous environments, Uspekhi Mat. Nauk 40 (1985), no. 2(242), 61-120, 238.

[19] Ledrappier, F., Quelques propriétés des exposants caractéristiques, Ecole d'été de SaintFlour 1982, Lecture Notes in Math., vol 1097, 305-396, Springer, 1984, Berlin.

[20] Marmi, S., Moussa, P., Yoccoz, J.-C, The cohomological equation for Roth-type interval exchange maps. J. Amer. Math. Soc. 18 (2005), no. 4, 823-872.

[21] Oseledec, V., A multiplicative ergodic theorem. Characteristic Ljapunov exponents of dynamical systems, Trudy Moskov. Mat. Obšč. 19 (1968), 179-210. 
[22] Peterson, J., Ph-D thesis, forthcoming (2008).

[23] Raugi, A., Théorème ergodique multiplicatif. Produits de matrices aléatoires indépendantes, Fascicule de probabilités, Univ. Rennes I, 1997, p. 43.

[24] Roitershtein, A., Transient random walks on a strip in a random environment, preprint 2006, to appear in Annals of Probability.

[25] SinaI, Y., The limit behaviour of a one-dimensional random walk in a random environment, Teor. Veroyatnost. iPrimenen. 27 (1982), num. 2, 247-258.

[26] Solomon, F., Random walks in a random environment, Ann. Probability 3 (1975), 1-31.

[27] Sznitman, A.-S., Topics in Random Walks in Random Environment, lecture given at the School and Conference on Probability Theory, Trieste, May 2002.

[28] Zeitouni, O., Random walks in random environment, Lectures on probability theory and statistics, Ecole d'Eté de probabilités de Saint-Flour XXXI - 2001. Springer : Berlin. Lect. Notes Math. 1837, 191-312 (2004).

Laboratoire d'Analyse et de Mathématiques Appliquées, Université Paris Xit, Faculté des Sciences et Technologies, 61 avenue du Général de Gaulle, 94010 Créteil Cedex, FRAnCE E-mail address : bremont@univ-paris12.fr 